\begin{tabular}{c} 
International Journal of Engineering \& Technology, $7(3.33)(2018) 115-119$ \\
International Journal of Engineering \& Technology \\
SPC \\
Website: www.sciencepubco.com/index.php/IJET \\
Research paper \\
\hline
\end{tabular}

\title{
Deep Convolution Neural Networks for Medical Image Analysis
}

\author{
Myung Jae Lim ${ }^{1}$, Da Eun Kim², Dong Kun Chung ${ }^{3}$, Hoon Lim ${ }^{4}$, Young Man Kwon ${ }^{3 *}$ \\ ${ }^{I}$ Department of Medical IT, College of Healthy Industry, Eulji University, Korea \\ e-mail:lk04@eulji.ac.kr \\ ${ }^{2}$ Department of Medical IT, College of Healthy Industry, Eulji University, Korea \\ e-mail : kdo10000@naver.com \\ ${ }^{3}$ Department of Medical IT, College of Healthy Industry, Eulji University, Korea \\ e-mail :ymkwon@eulji.ac.kr \\ ${ }^{4}$ Fine- tech water CO.,LTD \\ e-mail:hmbio@choi.com \\ *Corresponding author E-mail: ymkwon@eulji.ac.kr
}

\begin{abstract}
Breast cancer is a highly contagious disease that has killed many people all over the world. It can be fully recovered from early detection. To enable the early detection of the breast cancer, it is very important to classify accurately whether it is breast cancer or not. Recently, the deep learning approach method on the medical images such as these histopathologic images of the breast cancer is showing higher level of accuracy and efficiency compared to the conventional methods. In this paper, the breast cancer histopathological image that is difficult to be distinguished was analyzed visually. And among the deep learning algorithms, the CNN(Convolutional Neural Network) specialized for the image was used to perform comparative analysis on whether it is breast cancer or not. Among the CNN algorithms, VGG16 and InceptionV3 were used, and transfer learning was used for the effective application of these algorithms.

The data used in this paper is breast cancer histopathological image dataset classifying the benign and malignant of BreakHis. In the 2class classification task, InceptionV3 achieved 98\% accuracy. It is expected that this deep learning approach method will support the development of disease diagnosis through medical images.
\end{abstract}

Keywords: Deep Learning, Convolutional Neural Network, Medical Image, Breast Cancer, GoogLeNet, VGGNet

\section{Introduction}

In 2012, 1.7 million women were diagnosed with breast cancer worldwide. It is also ranked No. 2 in the number of deaths among female cancer patients, and in 2002, approximately 520,000 female patients died from breast cancer worldwide [1]. The best treatment method for breast cancer is early detection, which can be recovered completely from early detection. Therefore, it is very important to classify accurately whether it is breast cancer or not to enable early detection of breast cancer. In this process of using medical images for diagnosis, there can be different analysis results on the image depending on the medical laboratory technologists. However, this kind of error does not occur in the diagnosis method using the computer, and it can derive consistent diagnosis result [2]. There were numerous studies conducted on the machine learning method through the computer to enable accurate diagnosis of these medical images. Recently, the deep learning method of analysis is showing more outstanding results compared to the conventional machine learning methods, and is being applied to various fields including extraction and division of organs or cancer sites, or on image classification and disease recognition, etc [3, 27, 28]. The analysis and diagnosis of medical images uses histopathology images for the following reasons. The purpose is to study the abnormalities of diseased tissue and cell changes in comparison with healthy tissue and cells for electron microscope observation. In this paper, the deep learning approach method is applied to the breast cancer histopathology image of BreakHis to perform the task of classifying the image into benign and malignant. This method shows higher level of reliability and accuracy compared to conventional classification methods. The classifiers used in the classification of breast cancer cells are the VGG16 and InceptionV3 models that are $\mathrm{CNN}$ (Convolutional Neural Network) types used actively in the image classification on deep learning. Breast cancer histopathological image will be analyzed visually, and comparative analysis will be performed on its performance through the VGG16 and InceptionV3 models. In addition, to apply the algorithm shown above, the pre-trained neural network will be used for transfer learning. Two of the transfer learning methods will be used in this thesis. One method is to retrain only the last classification layer and the other is to readjust the overall pre-trained network [4]. In this thesis, the last layer learning method is called custom layer method because it changes the way layers are applied. All two transfer learning methods will be used to verify which transfer learning method is most useful for each algorithm. In conventional studies, the breast cancer image was used to apply the CNN models into both the overall layer learning method and the last layer learning method for study [5]. Also, there are many cases of application in not only limiting to the breast cancer images, but also to various pathological images including numerous cancer cells, gastro scope, CT, MRI, etc [2, 
6]. In this paper, the CNN model not used in the previous mentioned studies was applied additionally, and the layer used in transfer learning was applied differently to analyze the breast cancer image.

\section{Related Work}

\subsection{CNN}

CNN(Convolutional Neural Network) is a deep learning method that shows outstanding results in image recognition [24, 26]. In addition, the characteristics of $\mathrm{CNN}$ is that the convolutional layer and pooling layer are used, and ReLU (Rectified Linear Unit) is used for the activation function $[7,25]$. In the $\mathrm{CNN}$ model, there are many types such as VGGNet and Inception models that were used in this paper.

\subsubsection{VGGNet}

The VGGNet is a model that ranked second in the 2014 ImageNet Challenge classification sector, and the characteristic is to increase the structural depth through very small $3 \mathrm{X} 3$ convolutional filters. In this process, stride $1,3 \mathrm{X} 3$ convolution, max pooling and average pooling were used, and representative types is VGG16. It is a model that shows good results through simple structure [8].

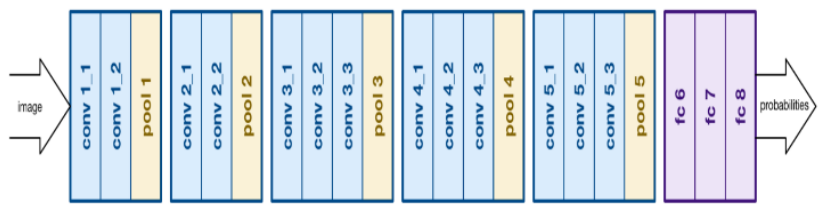

Fig. 1: The architecture of VGGNet[9]

\subsubsection{GoogLeNet}

GoogLeNet was ranked first in the 2014 ImageNet Challenge classification sector, and it was designed to use the Network in Network (NIN) structure to enable better extraction of the feature inside the local receptive field. Therefore, it is a model that reduces the filter size from $7 \times 7$ to $3 \mathrm{X} 3$, and is applied with the Batch normalization on the last fully connected layer. Representative types is InceptionV3 [10].

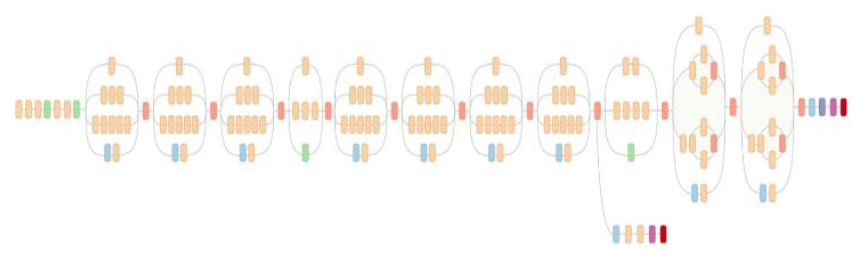

Fig. 2: The architecture of GoogLeNet[11]

\subsection{Gradient Descent Optimization Algorithms}

Gradient descent is used to control the weight of a neural network. This is a method of using gradient to minimize the value of loss function that defines the difference between the results produced in the network and the actual results. Gradient descent gradient in size as well as a constant in the opposite direction of the repeated loss to minimize the value of function. Function (1) is as follows.

$$
\theta=\theta-\eta \nabla_{\theta} J(\theta)
$$

The use of the entire set of traffic when calculating the Loss Function is called Batch Gradient Delay. However, this calculation requires too much calculation because the Loss Function is calculated for the entire data in one step. To prevent this, a method commonly referred to as Strategic Gradient Descent (SGD) is used.
There are various kinds of optimization algorithms, such as Adag$\mathrm{rad}$, Momentum, and so on. In this paper, there are RMSProp and Adam. The parameters of the network are $\theta, J(\theta)$ is loss function, $\nabla \theta \mathrm{J}(\theta)$ is gradient, $\eta$ is step size, $\gamma$ is momentum term, $\mathrm{t}$ is time step, $\epsilon$ is a small value to prevent dividing by zero, and Gt is a kdemension vector $[23,29]$

\subsubsection{RMSprop}

RMSProp(2) is a method proposed by Deep Learning's master Jeffrey Hinton to address Adagrad's shortcomings. It is a method that replaces the Gt obtained by converting the sum of the squared gradients from Adagrad's equation to an index average. This substitution allows the Gt to grow indefinitely, but maintain a relative size difference between variables in recent changes, such as Adagrad. In the following manner [23].

$G_{t}=\gamma G_{t-1}+(1-\gamma) g_{t}^{2}$
$\theta_{t}=\theta_{t-1}-\frac{\eta}{\sqrt{G_{t}+\epsilon}} g_{t}$

\subsubsection{Adam}

Adam(3) is an algorithm that looks like a combination of RMSProp and Momentum. And this is the most commonly used algorithm as the default. This method stores the index mean of the slopes, which have been calculated similarly to the Momentum method, and the index average of the squared values of the slopes, similar to RMSProp $[23,30]$.

$m_{t}=\beta_{1} m_{t-1}+\left(1-\beta_{1}\right) \nabla_{\theta} J(\theta)$

$v_{t}=\beta_{2} v_{t-1}+\left(1-\beta_{2}\right)\left(\nabla_{\theta} J(\theta)\right)^{2}$

\subsection{Transfer Learning}

A large-scale data set is required to enable deep neural network learning, and transfer learning using a model learned from another large-scale data set can be applied to solve the problem of data shortage. The transfer learning does not enable learning from scratch on the CNN, but is a method of applying the pre-trained model through the large-scale dataset to the data for actual learning. By using the transfer learning, it is possible to enable learning through small amount of data while avoiding over-fitting. There are three transfer learning methods as follows. The first method is using ConvNet as fixed feature extractor. Next is fine-tuning the ConvNet. Last one is using pretrained models. This paper will discuss second method $[4,12,13,14]$.

\subsubsection{Fine-Tuning the ConvNet}

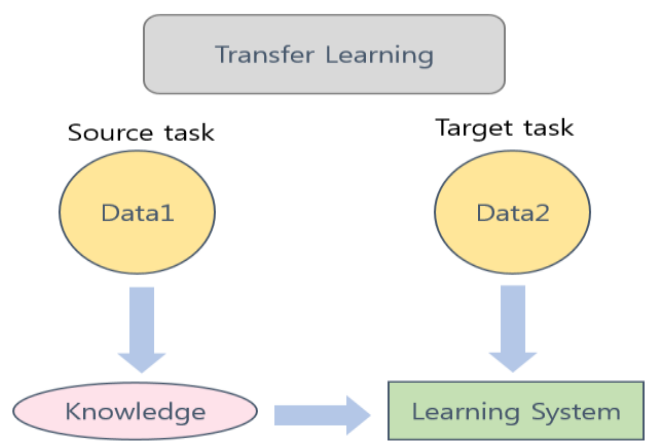

Fig. 3: The Overall flow of transfer learning

Fine-tuning is to not only replace and retrain the classifier on top of the ConvNet on the new dataset, but to also fine-tune the weights of the pretrained network by continuing the backpropaga- 
tion. It is possible to fine-tune all the layers of the ConvNet, or it's possible to keep some of the earlier layers fixed and only fine-tune some higher-level portion of the network. There are four main ways to do fine-tuning.

First, New dataset is small and similar to original dataset. Since the data is small, overfitting can be occurred. In this case, learn the linear classifier using CNN codes. Second, New dataset is large and similar to the original dataset. Since overfiting will not occur due to sufficient data, the entire network must be fine-tuning. Third, New dataset is small but very different from the original dataset. Because the data to be learned is different from the original data, the value of activation in the front layer of the network should be used to learn SVMs. Forth, New dataset is large and very different from the original dataset. Because of its large data, CNN may be deployed from the start, but it is also better to use a pretrained model. Fine-tuning the entire network since there is plenty of data. [4].

\section{Analysis Method}

\subsection{System Diagram}

The overall process of the system is shown as follows. The breast cancer image is inputted and passed through the image processing of Data augmentation and data pre-processing to derive the output.

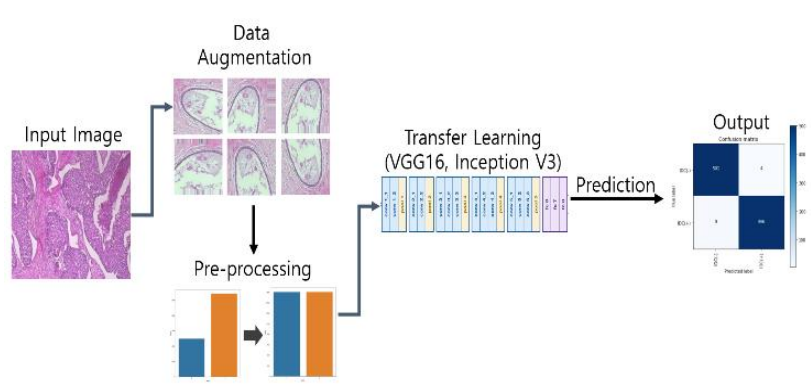

Fig. 4: Overall system diagram for classification

\subsection{Dataset}

Table 1: The Composition of the BreakHis Dataset used in the thesis

\begin{tabular}{|c|c|c|c|}
\hline Magnification & Benign & Malignant & Total \\
\hline $40 \mathrm{X}$ & 652 & 1,370 & 1,995 \\
\hline $100 \mathrm{X}$ & 644 & 1,437 & 2,081 \\
\hline $200 \mathrm{X}$ & 623 & 1,390 & 2,013 \\
\hline $400 \mathrm{X}$ & 588 & 1,232 & 1,820 \\
\hline Total of Images & 2,480 & 5,429 & 7,909 \\
\hline
\end{tabular}

In this paper, BreakHis (The Breast Cancer Histopathological Images) dataset was used. The BreakHis is composed of 9,109 microscopic images of breast tumor tissue $(2,480$ benign and 5,429 malignant) collected from 82 patients.

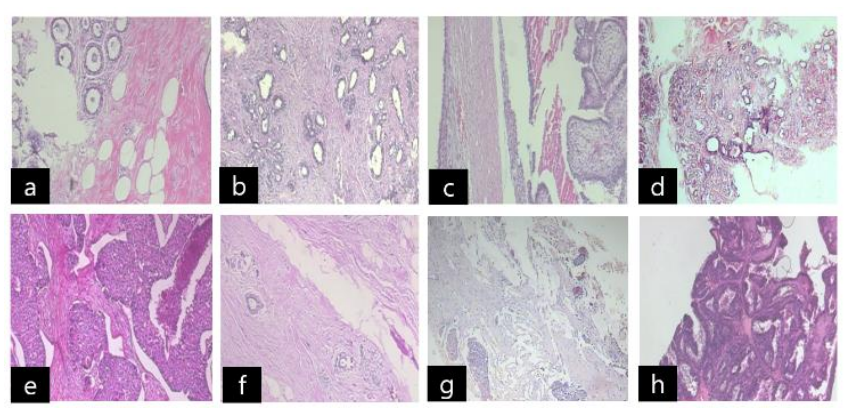

Fig.5: BreakHis dataset composed of benign and malignant images : benign [(a) : adenosis, (b) : fibroadenoma, (c) : phyllodes tumor, (d) : tubular adenoma], malignant [(e) : ductal carcinoma, (f) : lobular carcinoma, (g) : mucinous carcinoma, (h) : papillary carcinoma]
All image slides use $740 \mathrm{X} 460$ pixels, and different expansion ratios of 40X, 100X, 200X and 400X [16]. Figure 1 shows the histopathological image according to each classification.

\subsection{Data Augmentation}

In the process of Deep Neural Network learning, over-fitting of inhibiting the classification performance is occurred when there is insufficient number of required dataset, and to solve this issue, data augmentation is used to increase the number of learning images through the method of cutting or rotating the existing dataset. In this paper, the data augmentation was applied to classify the breast cancer histopathological image into benign and malignant effectively $[5,17,18]$. The rotation range was applied with 50 to enable random rotation of the image within 50 degrees, and 0.5 was applied both for the width shift range and the height shift range to move it randomly in horizontal and vertical directions within $50 \%$ of the total area. In addition, the horizontal flip and the vertical flip were applied with true to enable random flip in horizontal and vertical directions. The zoom was applied in the range of 0.5 .

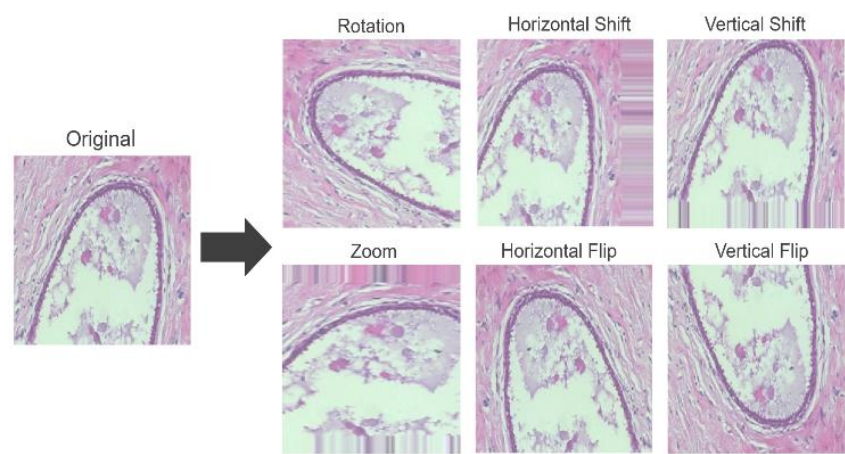

Fig. 6: Examples of methods applied to BreakHis dataset in this thesis : rotation, horizontal shift, vertical shift, zoom, horizontal flip, vertical flip

\subsection{Data Pre-Processing}

The color of the dataset was applied with RGB to preserve the tissue structures and different features of the histopathological image [5]. The image was resized into 224X224X3 or 299X299X3 to apply it appropriate for the VGG16 and InceptionV3 structures used in this paper $[8,10]$. In terms of BreakHis dataset used in this paper, there are 2,480 Benign images and 5,429 malignant images, so there is a great difference in the number of data included in each category. Data Imbalance can reduce the performance of $\mathrm{CNN}$, and to solve this issue, the Under-Sampling method was applied. Based on the determined rule, the number of data of many classes is sampled by the number of data of a small number of classes [19].

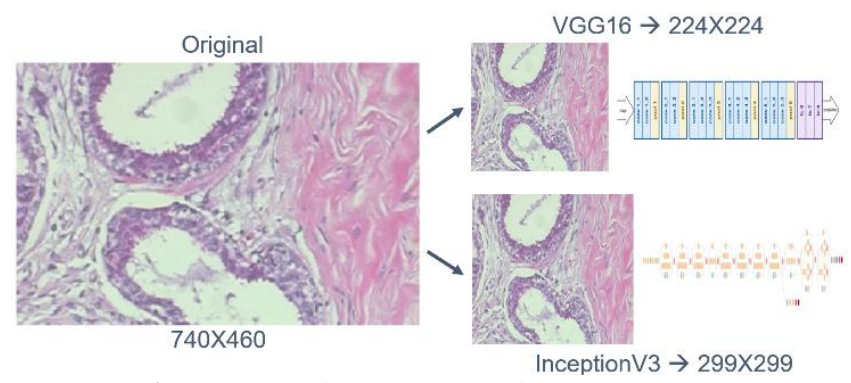

Fig.7: Resizes of original images for each model

\subsection{Training}

After data pre-processing and data argumentation were completed, fine-tuning was enabled on the CNN model to perform training on the breast cancer histopathological image (Breakhis dataset). The 
CNN models used in the training were Vgg16 and InceptionV3, and pre-trained network was applied to each model. In addition, the fine-tuning was performed by using the pre-trained model trained through the large-scale data of the ImageNet. In this experiment, pretrained network was applied and the last layer was omitted. Because we added a custom classification network on the top of the prebuilt network and replace the last layer. The layers used in the custom network are GlobalAveragePooling(GAP) and two Fully Connected Layer(FC). Global Average Pooling is a method used to replace traditional Fully Connected Layer on CNN. There is a tendency to overcompose at Fully Connected Layers when using CNN. Dropout was proposed as a regularizer by Hilton et al, which contributed to the prevention of overcongestion [31]. Fully Connected Layer is

Then only the custom layer was learned, or the whole layer was learned $[4,5,15,20]$.

In the process of training the models in this paper, optimizer appropriate for each algorithm was used. The Vgg16 was applied with Adam optimizer $[8,21]$ and the InceptionV3 was applied with RMSprop optimizer [10].

For the learning rate, 0.0001 was applied, along with $1 \mathrm{e}-6$ for decay, 32 for batch size, and 100 for epoch. Among the overall images, $20 \%$ for test set and $80 \%$ for train set were set-up.

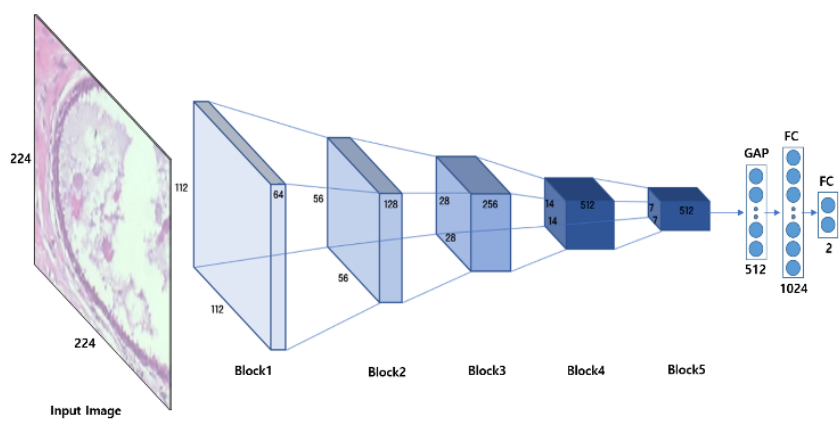

Fig. 8: Overall network architecture for transfer learning VGG16

\section{Result}

Fine-tuning was performed on the breast cancer dataset to classify into Malignant and Benign, and among the fine-tuning methods, two methods of learning only the custom layer and learning whole layers were used. The values shown in Table 1 can be described as follows: accuracy(ACC), sensitivity(SE), specificity(SP), preci$\operatorname{sion}(\mathrm{P})[22]$.

Table 2: The result of two methods of fine-tuning applied to each model

\begin{tabular}{|c|l|c|c|c|c|}
\hline Model name & Layer type & ACC & SE & SP & P \\
\hline VGG16 & Custom & 0.83 & 0.82 & 0.85 & 0.84 \\
\hline VGG16 & Whole & 0.97 & 0.96 & 0.98 & 0.97 \\
\hline InceptionV3 & Custom & 0.74 & 0.71 & 0.79 & 0.76 \\
\hline InceptionV3 & Whole & 0.98 & 0.98 & 0.98 & 0.99 \\
\hline
\end{tabular}

\section{Conclusion and Future Work}

In this paper, breast cancer image analysis was performed to analyze the most fatal cancer in women. It was enabled through the deep learning method for the effective and accurate diagnosis of breast cancer. Among the deep learning methods, CNN algorithm types of Vgg16 and InceptionV3 were used to focus on classifying whether it is a breast cancer cell or not. In addition, to enhance the performance of the classifier, pre-trained network from the ImageNet was used to perform Transfer Learning.

From the experiment, the following results were confirmed. There was difference between VGG16 and InceptionV3. And whole layer method has higher performance than custom layer. If sufficient computing environment and professional medical knowledge are supported, future work will be a simpler and more accurate cancer diagnosis deep learning system. This can be applied to a variety of medical images and can greatly assist medical image diagnosis. The vast amount of data can be used to help doctors make decisions and to help many people by acting as doctors in poor countries without doctors. Deep Learning will soon be able to conquer diseases such as cancer, providing the best diagnosis and customized treatment anywhere.

\section{Acknowledgement}

The project has received funding from the Fine tech water CO., Ltd. The project has been possible due to the collaboration of Fine tech water CO., Ltd and Seoungnam Senior Experience Complex 's staff and students as well as many other institutions, companies, individuals and colleagues.

\section{References}

[1] "Latest world cancer statistics", World Health Organization, 2013

[2] Jeonghun Yoon, YoungJu Jeong, Giwon Lee, Seongju Ryu, Noa Seo, Jihun Yoon, Jihun Noh, Jugyeon Lim, Donggyun Kim, “A Study on the Estimation of pathology Result from Gastroscopy Image Using Deep Learning”, Korean Department of Internal Medicine Association, 2017

[3] Hansang Lee, Minseok Park, Junmo Kim, "Deep Learning in Medical Image", Journal of Korean Medical Imaging, 2014

[4] http://cs231n.github.io/transfer-learning/

[5] Janowczyk A1, Madabhushi A1., "Breast Cancer Histopathological Image Classification: A Deep Learning Approach", doi: https://doi.org/10.1101/242818, 2018

[6] Jeong Won Min, Dong Joong Kang. "Deep Meta Learning Based Classification Problem Learning Method for Skeletal Maturity Indication." Journal of Korea Multimedia Society, 21.2, 2018

[7] G. E. Dahl, T. N. Sainath, and G. E. Hinton, "Improving deep neural networks for LVCSR using rectified linear units and dropout," IEEE International Conference on Acoustics, Speech and Signal Processing (ICASSP), 2013

[8] Karen Simonyan, Andrew Zisserman, "VERY DEEP CONVOLUTIONAL NETWORKS FOR LARGE-SCALE IMAGE RECOGNITION", arXiv:1409.1556v6 [cs.CV], 2015

[9] http://machinethink.net/blog/convolutional-neural-networks-on-theiphone-with-vggnet/

[10] Christian Szegedy, Vincent Vanhoucke, Sergey Ioffe, Jon Shlens, Zbigniew Wojna; "Rethinking the Inception Architecture for Computer Vision", The IEEE Conference on Computer Vision and Pattern Recognition (CVPR), pp. 2818-2826, 2016

[11] https://adeshpande3.github.io/The-9-Deep-Learning-Papers-YouNeed-To-Know-About. Htm

[12] Jeong Won Min, Dong Joong Kang. "Deep Meta Learning Based Classification Problem Learning Method for Skeletal Maturity Indication." Journal of Korea Multimedia Society, 21.2, 2018

[13] Dongjin Lee, Jaehong Kim, Han Seungjun, Choi Jeongdan, Cheong Hee Park. "Traffic light recognition using the LISA traffic light dataset.", Korean Industrial Engineering Association, 2016

[14] S. Pan and Q. Yang. "A survey on transfer learning. Knowledge and Data Engineering”, IEEE Transactions on, 22(10):1345-1359, 2010.

[15] https://keras.io/applications

[16] https://web.inf.ufpr.br/vri/databases/breast-cancerhistopathological-database-breakhis/

[17] Se-Ho Lim, Yong-Goo Shin, Cheol-Hwan Yoo, Han-Kyu Lee, Sung-Jea Ko. "Data Augmentation method using WGAN." Korean Electronic Engineering Society, 2017

[18] In-kyu Choi, Hyok Song, Sangyong Lee, Jisang Yoo. "Facial Expression Classification Using Deep Convolutional Neural Network." JOURNAL OF BROADCAST ENGINEERING, 22.2, 2017

[19] Pilseong Kang, Seongjun Cho, "EUS SVMs: Ensemble of UnderSampled SVMs for Data Imbalance Problems,“ Korean Industrial Engineering Association, 2006

[20] Antonio Guilli, “TensorFlow 1.x Deep Learning Cookbook”, 2017

[21] Jungbeom Lee, Sungroh Yoon. "Video Sequence Prediction using Optical Flow.", Korean Information Science Association, 2017

[22] M. Sokolova and G. Lapalme. A systematic analysis of performance measures for classification tasks. Inf. Process. Manage., 45(4):427-437, 2009 
[23] http://shuuki4.github.io/deep\%20learning/2016/05/20/GradientDescent-Algorithm-Overview.html

[24] Jaewan Yang, Youngdoo Lee, Insoo Koo, "Sensor Fault Detection Scheme based on Deep Learning and Support Vector Machine", The Journal of The Institute of Internet, Broadcasting and Communication, 2018

[25] Seokcheon Park, "Design and Implementation of Personal Information Identification and Masking System Based on Image Recognition", The Journal of The Institute of Internet, Broadcasting and Communication, 2017

[26] Youngdo Joo, "Drone Image Classification based on Convolutional Neural Networks", The Journal of The Institute of Internet, Broadcasting and Communication, 2017

[27] Minsoo Kang, Yonggyu Jung, Duhwan Jang, "A study on the Search of Optimal Aquaculture fram condition based on Machine Learning", The Journal of The Institute of Internet, Broadcasting and Communication, 2017

[28] Dohyeon Choi, Jungoh Park, "A study on Security Authentication Vector Generation of Virtualized Internal Environment using Machine Learning Algorithm”, The Journal of The Institute of Internet, Broadcasting and Communication, 2016

[29] Sebastian Ruder, "An overview of gradient descent optimization algorithms", http://arxiv.org/abs/1609.04747, 2016

[30] Diederik P. Kingma, Jimmy Lei Ba, "ADAM: A METHOD FOR STOCHASTIC OPTIMIZATION", a conference paper at the 3rd International Conference for Learning Representations, San Diego, 2015

[31] https://flonelin.wordpress.com/2017/12/22/global-average-pooling/ 